\title{
Productivity Analysis of Volume Fractured Vertical Well Model in Tight Oil Reservoirs
}

\author{
Jiahang Wang, ${ }^{1}$ Xiaodong Wang, ${ }^{1}$ Wenli Xu, ${ }^{1}$ Cheng Lu, ${ }^{1,2}$ \\ Wenxiu Dong, ${ }^{1}$ and Cong Zhang ${ }^{1,2}$ \\ ${ }^{1}$ China University of Geosciences, Beijing 100083, China \\ ${ }^{2}$ The Key Laboratory of Unconventional Petroleum Geology, CGS, Beijing 100029, China \\ Correspondence should be addressed to Cheng Lu; 442170010@qq.com
}

Received 18 October 2016; Accepted 27 December 2016; Published 26 January 2017

Academic Editor: Jian G. Zhou

Copyright (C) 2017 Jiahang Wang et al. This is an open access article distributed under the Creative Commons Attribution License, which permits unrestricted use, distribution, and reproduction in any medium, provided the original work is properly cited.

\begin{abstract}
This paper presents a semianalytical model to simulate the productivity of a volume fractured vertical well in tight oil reservoirs. In the proposed model, the reservoir is a composite system which contains two regions. The inner region is described as formation with finite conductivity hydraulic fracture network and the flow in fracture is assumed to be linear, while the outer region is simulated by the classical Warren-Root model where radial flow is applied. The transient rate is calculated, and flow patterns and characteristic flowing periods caused by volume fractured vertical well are analyzed. Combining the calculated results with actual production data at the decline stage shows a good fitting performance. Finally, the effects of some sensitive parameters on the type curves are also analyzed extensively. The results demonstrate that the effect of fracture length is more obvious than that of fracture conductivity on improving production in tight oil reservoirs. When the length and conductivity of main fracture are constant, the contribution of stimulated reservoir volume (SRV) to the cumulative oil production is not obvious. When the SRV is constant, the length of fracture should also be increased so as to improve the fracture penetration and well production.
\end{abstract}

\section{Introduction}

Reservoir stimulation technologies have been widely applied to develop tight oil reservoirs. Volume fracturing technique is one of these methods which has been widely used to improve the productivity of low permeability and tight reservoirs. After repeated acid fracturing treatment to the fractured brittle reservoirs, hydraulic fracture, natural fracture, and shear cracks are mutually staggered and form a certain stimulated zone of joint fracture network near the wellbore, which changes the flow pattern, reduces the flow resistance, and improves production of a single well [14]. Testing and evaluating fracture network reconstruction along the well and productivity behavior are essential for improving the performance of production well in tight reservoirs after volume fracturing. Rate decline analyzing is one of the key methodologies to estimate reservoir parameters, such as permeability, porosity, length, width, and skin factor.
Due to the advanced techniques in fracturing, the behavior of rate decline curves in a fractured well has attracted increasing attention recently. In terms of numerical simulation, Al-Salem et al. established the model by using the vertical and horizontal orthogonal crack network to approximately substitute volume reconstruction [5], and this model has been widely used since then [6-9]. Combining the micro seismic exploration results, Arvind approximately simulated the volume and the degree of the reconstruction region around wells [10]. However, Du et al. described the transformation region volume by using the Kazemi dual medium model [11]. In terms of the analytic model, Liu et al. $[12,13]$ and Lei and Gang [14] described the fracture distribution of volume transformation region of vertical wells by using the fractal theory, and the production of cold and heavy oil with carrying sand is studied based on their model. Recently, a composite reservoir model with fractal permeability was applied to evaluate the productivity of tight oil reservoirs [15-17]. 
Compared with the analytical method, the numerical simulation method is able to deal with the complicated seepage problem to a large extent by the grid division, while the procedure is complicated and causes large amount of computation resources. The fractal theory can describe the spatial distribution of fracture better, but it is not suitable for the pressure transmission behavior and the artificial fracture parameter optimization research. Liao and Chen described the pressure transient analysis of volume fracturing well without considering the wellbore storage effect and skin effect, and 5 flow regimes were recognized in the transient pressure type curves [17]. So far, there has not been an overall well test interpretation model for the vertical wells with stimulated volume in fractured tight reservoirs.

In this work, we propose a semianalytical model to simulate the productivity of volume fractured vertical well in a tight oil reservoir by Laplace transform and Stehfest numerical inversion [18]. By modeling the stimulated volume as the dual medium and coupling the stimulated formation with the discretized artificial fracture, a semianalytical solution is obtained. Finally the effects of some sensitive parameters, such as storage capacity ratio, crossflow coefficient, fracture conductivity, fracture length, stimulated volume, and skin factor on the type curve,s are also analyzed extensively.

\section{Mathematical Model}

A stimulated volume with joint network is formed near the wellbore in brittle tight reservoirs after repeated acid fracturing treatments. The stimulated fracture network can be normally subdivided into two parts, the inner artificial main fracture and the outer natural fractured zone, respectively. The fluid flow in the main fracture is linear and follows Darcy's law and the classical Warren-Root model [19] is used to describe the fracture distribution and seepage flow in the outer area where there are no artificial fractures and the permeability is very low $(<0.1 \mathrm{mD})$ because of low connectivity. Affected by the extension of the artificial fracture and the brittle shear of reservoir rock in outer area, the artificial fracture and natural fractures are arranged in a crisscross pattern and change the flow pattern mainly in fractures. In this work, the fluid supplied to the stimulated volume region by natural fractures is neglected since it is much smaller compared with that from the artificial main fracture [20, 21]. In summary, the model assumptions are listed below:

(1) The model is homogeneous and isotropic along the radial direction.

(2) The pressure is constant and both fluid and rock are slightly compressible.

(3) Fractures are the main flow channel, and the seepage flow is laminar and isothermal.

(4) The conductivity of vertical artificial fracture is finite and the fracture fully penetrated the formation with the height equal to the thickness of the reservoir.

Let us consider a volume fractured well in a circular closed reservoir. The main vertical fracture with finite conductivity has a half length $x_{\mathrm{f}}$, a width $b_{\mathrm{f}}$, and a permeability $k_{\mathrm{f}}$, and penetrates the formation vertically. The classical WarrenRoot model is used to simulate the microcracks produced by volume fracturing in the reservoir formation. The reservoir is composed of a fracture network and matrix blocks. The fracture network possesses a bulk fracture porosity $\phi_{2 f}$ and total compressibility $c_{2 \mathrm{f}}$. The matrix blocks are slabs of thickness $h$, permeability $k_{2 \mathrm{~m}}$, porosity $\phi_{2 \mathrm{~m}}$, and total compressibility $c_{2 \mathrm{~m}}$.

The fluid in the reservoir is slightly compressible and its viscosity is $\mu$. The flow process in the system can be studied by breaking up the medium into three parts and taking the interaction among different parts into account. These regions are hydraulic fracture in the inner area and reservoir fracture network and reservoir matrix in the outer area (see in Figure 1).

2.1. Region $I$. The flow within the hydraulic fracture is considered to be linear because the fracture width, $b_{\mathrm{f}}$, is much smaller than fracture length and fracture height. It is assumed that the fluid flow into the wellbore takes place only through the hydraulic fracture and the fluid flow from the reservoir into the hydraulic fracture occurs only through the reservoir fracture network because $k_{2 \mathrm{f}}$ is much larger than $k_{2 \mathrm{~m}}$. Figure 1 illustrates the characteristic of this model, and here $q_{\mathrm{f}}(x, t)$ is the flow rate going to the fracture per unit length.

Cinco et al. have demonstrated that the compressibility of the hydraulic fracture can be neglected for practical purposes because the fracture volume is very limited [22]. Hence the flow within the fracture can be considered to be incompressible. Under these conditions, the transient flow in the hydraulic fracture can be described by the following equations in terms of dimensionless variables:

$$
\frac{\partial^{2} p_{\mathrm{fD}}}{\partial x_{\mathrm{D}}^{2}}+\frac{2}{c_{\mathrm{fD}}} \frac{\partial p_{2 \mathrm{fD}}\left(y_{\mathrm{D}}, t_{\mathrm{D}}\right)}{\partial y_{\mathrm{D}}}=0, \quad 0<x_{\mathrm{D}}<1 .
$$

Inner boundary condition

$$
\frac{d p_{\mathrm{fD}}(0)}{d x_{\mathrm{D}}}=-\frac{\pi}{c_{\mathrm{fD}}} .
$$

Outer boundary condition

$$
\frac{d p_{\mathrm{fD}}(1)}{d x_{\mathrm{D}}}=0
$$

The flow correlation formula for surface of the fracture is

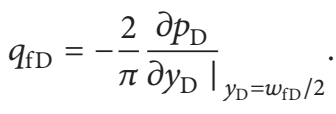

We can obtain the solution in Laplace domain through combining (1)-(4); that is,

$$
\begin{aligned}
p_{\mathrm{wD}}\left(x_{\mathrm{D}}\right)-p_{\mathrm{fD}}\left(x_{\mathrm{D}}\right) \\
=\frac{\pi}{c_{\mathrm{fD}}}\left(x_{\mathrm{D}}-\int_{0}^{x_{\mathrm{D}}} \int_{0}^{v} q_{\mathrm{fD}}\left(u, t_{\mathrm{D}}\right) d u d v\right),
\end{aligned}
$$



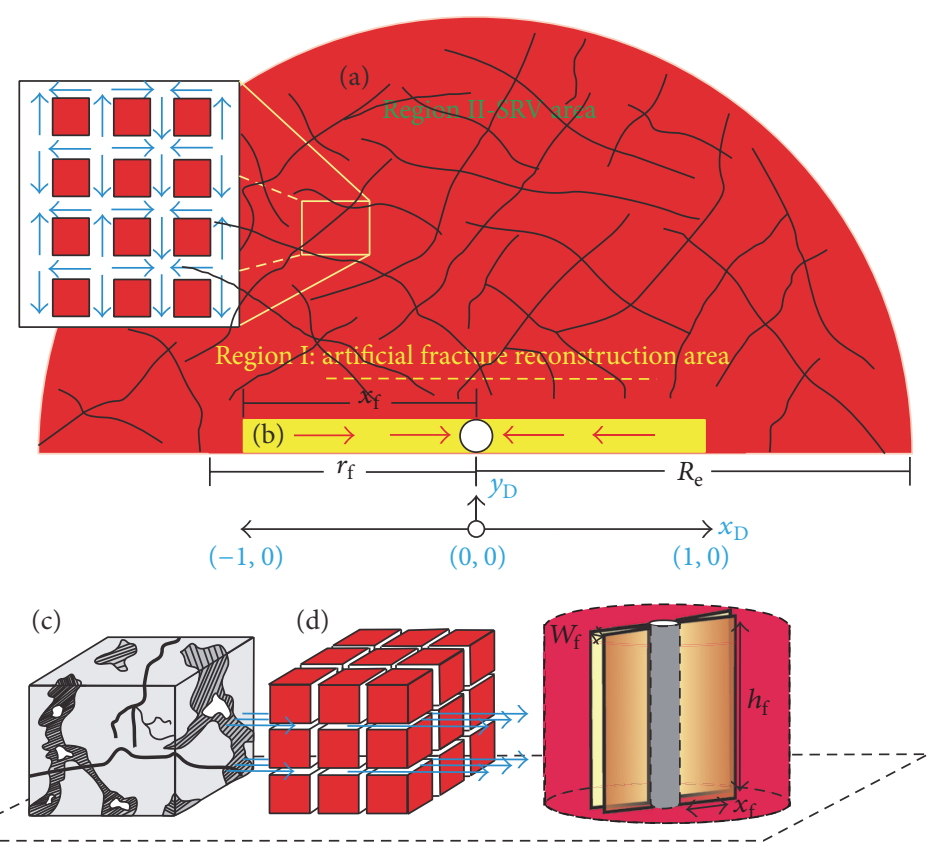

FiguRE 1: Schematic diagram: (a) stimulated reservoir volume model. (b) Physical modeling scheme of artificial main fracture. (c) The system of matrix. (d) Classic Warren-Root dual medium model.

where

$$
\begin{aligned}
P_{n \mathrm{D}} & =\frac{k_{2 \mathrm{f}} h\left(P_{i}-P\right)}{1.842 \times 10^{-3} q \mu B}, \quad n=\mathrm{f}, \mathrm{f} 2, \mathrm{~m} 2 ; \\
w_{\mathrm{D}} & =\frac{w_{\mathrm{f}}}{x_{\mathrm{f}}} ; \\
x_{\mathrm{D}} & =\frac{x}{x_{\mathrm{f}}} ; \\
y_{\mathrm{D}} & =\frac{y}{x_{\mathrm{f}}} ; \\
c_{\mathrm{fD}} & =\frac{k_{\mathrm{f}} w_{\mathrm{f}}}{k_{2 \mathrm{f}} x_{\mathrm{f}}} ; \\
t_{\mathrm{D}} & =\frac{3.6 \eta_{\omega} t}{x_{\mathrm{f}}^{2}} ; \\
\eta_{\omega} & =\frac{k_{2 \mathrm{f}}}{\mu\left(\phi_{2 \mathrm{f}} c_{2 \mathrm{f}}+\phi_{2 \mathrm{~m}} c_{2 \mathrm{~m}}\right)} ; \\
\lambda_{2} & =\frac{(\phi c)_{2 \mathrm{f}}}{(\phi c)_{2 \mathrm{f}}+(\phi c)_{2 \mathrm{~m}}} ; \\
k_{2 \mathrm{~m}} x_{\mathrm{f}}^{2} & k_{2 \mathrm{f}}
\end{aligned}
$$

2.2. Region II. As mentioned above, the stimulated formation is full of microcracks. The reservoir is represented by a fracture network and matrix blocks. It is assumed that the characteristics of both the fracture network and matrix blocks remain constant. The flow from the reservoir into the hydraulic fracture only occurs through the fracture network, as generally considered in the literatures for dual medium reservoirs.

The transient flow in the formation can be described by

$$
\frac{\partial^{2} \widetilde{p}_{2 \mathrm{fD}}}{\partial r_{\mathrm{D}}^{2}}+\frac{1}{r_{\mathrm{D}}} \frac{\partial \widetilde{p}_{2 \mathrm{fD}}}{\partial r_{\mathrm{D}}}=s \widetilde{p}_{2 \mathrm{fD}}-\widetilde{q}_{2 \mathrm{mD}}
$$

because

$$
\begin{aligned}
q & =-\left(V \phi c_{t}\right)_{2 \mathrm{~m}} \frac{\partial P_{2 \mathrm{~m}}}{\partial t}=\frac{k_{2 \mathrm{~m}} A_{2 \mathrm{~m}}}{\mu l V}\left(P_{2 \mathrm{~m}}-P_{2 \mathrm{f}}\right) \\
& =\frac{k_{2 \mathrm{~m}}}{\mu l^{2}}\left(P_{2 \mathrm{~m}}-P_{2 \mathrm{f}}\right) .
\end{aligned}
$$

We can get the equation below:

$$
q_{2 \mathrm{mD}}=\lambda_{2}\left(P_{2 \mathrm{fD}}-P_{2 \mathrm{mD}}\right)=\left(1-\omega_{2}\right) \frac{\partial P_{2 \mathrm{mD}}}{\partial t_{\mathrm{D} \omega}} .
$$

Equation (7) can be further simplified:

$$
\frac{\partial^{2} \widetilde{p}_{2 \mathrm{fD}}}{\partial r_{\mathrm{D}}^{2}}+\frac{1}{r_{\mathrm{D}}} \frac{\partial \widetilde{p}_{2 \mathrm{fD}}}{\partial r_{\mathrm{D}}}=s f_{2}(s) \widetilde{p}_{2 \mathrm{fD}} .
$$

Inner boundary condition

$$
\left[r_{\mathrm{D}} \frac{\partial \widetilde{P}_{2 \mathrm{fD}}}{\partial r_{\mathrm{D}}}\right]_{r_{\mathrm{D}}=0}=-\frac{q_{\mathrm{f}}}{q}=-\widetilde{q}_{\mathrm{fD}} .
$$

Outer boundary condition

$$
\left[r_{\mathrm{D}} \frac{\partial \widetilde{P}_{2 \mathrm{fD}}}{\partial r_{\mathrm{D}}}\right]_{r_{\mathrm{D}}=r_{\mathrm{eD}}}=0
$$


where

$$
f_{2}(s)=\frac{\omega_{2}\left(1-\omega_{2}\right) s+\lambda_{2}}{\left(1-\omega_{2}\right) s+\lambda_{2}} .
$$

Combining Equations (7)-(12) can obtain the point source solution of formation transient flow:

$$
\begin{aligned}
\tilde{p}_{2 \mathrm{fD}}= & \tilde{q}_{\mathrm{fD}}(s) \\
& \cdot\left[\frac{K_{1}\left(r_{\mathrm{eD}} \sqrt{z}\right)}{I_{1}\left(r_{\mathrm{eD}} \sqrt{z}\right)} I_{0}\left(r_{\mathrm{D}} \sqrt{z}\right)+K_{0}\left(r_{\mathrm{D}} \sqrt{z}\right)\right],
\end{aligned}
$$

where

$$
z=s \frac{\omega_{2}\left(1-\omega_{2}\right) s+\lambda_{2}}{\left(1-\omega_{2}\right) s+\lambda_{2}}=s f_{2}(s) .
$$

Further, the solution of plane source is obtained by integrating point source in terms of Bessel functions. The pressure distribution of this system is then given below

$$
\begin{aligned}
& \widetilde{p}_{2 \mathrm{fD}}\left(x_{\mathrm{D}}, 0, s\right)=\frac{1}{2} \\
& \cdot \int_{-1}^{1} \widetilde{q}_{\mathrm{fD}}\left[\frac{K_{1}\left(r_{\mathrm{eD}} \sqrt{z}\right)}{I_{1}\left(r_{\mathrm{eD}} \sqrt{z}\right)} I_{0}\left\{\left[\left(x_{\mathrm{D}}-\sigma\right)^{2}\right]^{1 / 2} \sqrt{z}\right\}\right. \\
& \left.+K_{0}\left\{\left[\left(x_{\mathrm{D}}-\sigma\right)^{2}\right]^{1 / 2} \sqrt{z}\right\}\right] d \sigma .
\end{aligned}
$$

Combining Equations (5) and (16), we can obtain

$$
\begin{gathered}
\tilde{p}_{\mathrm{wD}}(s)=\frac{\pi}{s c_{\mathrm{fD}}}\left(x_{\mathrm{D}}-s \int_{0}^{x_{\mathrm{D}}} \int_{0}^{v} \widetilde{q}_{\mathrm{fD}}(u) d u d v\right)+\frac{1}{2} \\
\cdot \int_{-1}^{1} \widetilde{q}_{\mathrm{fD}}\left[\frac{K_{1}\left(r_{\mathrm{eD}} \sqrt{z}\right)}{I_{1}\left(r_{\mathrm{eD}} \sqrt{z}\right)} I_{0}\left\{\left[\left(x_{\mathrm{D}}-\sigma\right)^{2}\right]^{1 / 2} \sqrt{z}\right\}\right. \\
\left.+K_{0}\left\{\left[\left(x_{\mathrm{D}}-\sigma\right)^{2}\right]^{1 / 2} \sqrt{z}\right\}\right] d \sigma .
\end{gathered}
$$

Considering the fracture symmetry,

$$
\tilde{q}_{\mathrm{fD}}(\sigma, s)=\tilde{q}_{\mathrm{fD}}(-\sigma, s) .
$$

Equation (17) becomes

$$
\begin{aligned}
& \widetilde{p}_{\mathrm{wD}}(s)-\frac{\pi}{s c_{\mathrm{fD}}}\left(x_{\mathrm{D}}-s \int_{0}^{x_{\mathrm{D}}} \int_{0}^{v} \tilde{q}_{\mathrm{fD}}(u) d u d v\right)=\frac{1}{2} \\
& \cdot \int_{0}^{1} \widetilde{q}_{\mathrm{fD}}\left[\frac { K _ { 1 } ( r _ { \mathrm { eD } } \sqrt { z } ) } { I _ { 1 } ( r _ { \mathrm { eD } } \sqrt { z } ) } \left\{I_{0}((x-\sigma) \sqrt{z})\right.\right. \\
& \left.+I_{0}((x+\sigma) \sqrt{z})\right\}+\left\{K_{0}((x-\sigma) \sqrt{z})\right. \\
& \left.\left.+K_{0}((x+\sigma) \sqrt{z})\right\}\right] d \sigma .
\end{aligned}
$$

Equation (19) gives the transient solution of the finite conductivity vertical fracture.
2.3. Solution. Assuming the fracture can be divided into $n$ segments, the first part on the right side of (19) can be written as

$$
\begin{aligned}
& \frac{1}{2} \frac{K_{1}\left(r_{\mathrm{eD}} \sqrt{z}\right)}{I_{1}\left(r_{\mathrm{eD}} \sqrt{z}\right)} \\
& \quad \cdot \int_{0}^{1} \widetilde{q}_{\mathrm{fD}}\left\{I_{0}((x-\sigma) \sqrt{z})+I_{0}((x+\sigma) \sqrt{z})\right\} d \sigma \\
& \quad=\frac{1}{2} \frac{K_{1}\left(r_{\mathrm{eD}} \sqrt{z}\right)}{I_{1}\left(r_{\mathrm{eD}} \sqrt{z}\right)} \times \sum_{i=1}^{n} \widetilde{q}_{\mathrm{fD}}(\sigma, s) \\
& \quad \cdot \int_{x_{\mathrm{D} i-1}}^{x_{\mathrm{D} i}}\left\{I_{0}((x-\sigma) \sqrt{z})+I_{0}((x+\sigma) \sqrt{z})\right\} d \sigma
\end{aligned}
$$

and the second part can be expressed as

$$
\begin{aligned}
& \frac{1}{2} \int_{0}^{1} \tilde{q}_{\mathrm{fD}}\left\{K_{0}((x-\sigma) \sqrt{z})+K_{0}((x+\sigma) \sqrt{z})\right\} d \sigma \\
& \quad=\frac{1}{2} \times \sum_{i=1}^{n} \tilde{q}_{\mathrm{fD}}(\sigma, s) \\
& \quad \cdot \int_{x_{\mathrm{D} i-1}}^{x_{\mathrm{D} i}}\left\{K_{0}((x-\sigma) \sqrt{z})+K_{0}((x+\sigma) \sqrt{z})\right\} d \sigma .
\end{aligned}
$$

By using variable substitution method, the integral form of $K_{0}$ and $I_{0}$ can be expressed as follows:

$$
\begin{aligned}
& \int_{-1}^{1} K_{0}\left[\sqrt{(x-\alpha)^{2}} \varepsilon_{n}\right] d \alpha \\
& =\frac{1}{\varepsilon_{n}}\left\{\pi-\left[K i_{1}\left(\varepsilon_{n}+\varepsilon_{n} x_{\mathrm{D}}\right)+K i_{1}\left(\varepsilon_{n}-\varepsilon_{n} x_{\mathrm{D}}\right)\right]\right\}, \\
& \int_{-1}^{1} I_{0}\left[\sqrt{(x-\alpha)^{2}} \varepsilon_{n}\right] d \alpha \\
& =\frac{1}{\varepsilon_{n}}\left[I i_{1}\left(\varepsilon_{n}+\varepsilon_{n} x_{\mathrm{D}}\right)+I i_{1}\left(\varepsilon_{n}-\varepsilon_{n} x_{\mathrm{D}}\right)\right],
\end{aligned}
$$

where

$$
\begin{gathered}
K i_{1}(x)=\int_{x}^{\infty} K_{0}(t) d t \\
I i_{1}(x)=\int_{\infty}^{x} I_{0}(t) d t .
\end{gathered}
$$

(1) If $j>i$,

$$
\begin{aligned}
& \int_{x_{\mathrm{D} i-1}}^{x_{\mathrm{D} i}} K_{0}\left[\left(x_{\mathrm{D} j}-\sigma\right) \sqrt{z}\right] d \sigma \\
& \quad=\frac{1}{\sqrt{z}}\left\{K i_{1}\left[\left(x_{\mathrm{D} j}-x_{\mathrm{D} i}\right) \sqrt{z}\right]\right. \\
& \left.\quad-K i_{1}\left[\left(x_{\mathrm{D} j}-x_{\mathrm{D} i-1}\right) \sqrt{z}\right]\right\}=a(j, i),
\end{aligned}
$$




$$
\begin{aligned}
& \int_{x_{\mathrm{D} i-1}}^{x_{\mathrm{D} i}} I_{0}\left[\left(x_{\mathrm{D} j}-\sigma\right) \sqrt{z}\right] d \sigma \\
& \quad=\frac{1}{\sqrt{z}}\left\{I i_{1}\left[\left(x_{\mathrm{D} j}-x_{\mathrm{D} i}\right) \sqrt{z}\right]\right. \\
& \left.\quad-I i_{1}\left[\left(x_{\mathrm{D} j}-x_{\mathrm{D} i-1}\right) \sqrt{z}\right]\right\}=b(j, i) .
\end{aligned}
$$

(2) If $j<i$,

$$
\begin{aligned}
& \int_{x_{\mathrm{D} i-1}}^{x_{\mathrm{D} i}} K_{0}\left[\left(x_{\mathrm{D} j}-\sigma\right) \sqrt{z}\right] d \sigma \\
& =\frac{1}{\sqrt{z}}\left\{K i_{1}\left[\left(x_{\mathrm{D} i-1}-x_{\mathrm{D} j}\right) \sqrt{z}\right]\right. \\
& \left.-K i_{1}\left[\left(x_{\mathrm{D} i}-x_{\mathrm{D} j}\right) \sqrt{z}\right]\right\}=a(j, i), \\
& \int_{x_{\mathrm{D} i-1}}^{x_{\mathrm{D} i}} I_{0}\left[\left(x_{\mathrm{D} j}-\sigma\right) \sqrt{z}\right] d \sigma \\
& =\frac{1}{\sqrt{z}}\left\{I i_{1}\left[\left(x_{\mathrm{D} i-1}-x_{\mathrm{D} j}\right) \sqrt{z}\right]\right. \\
& \left.-I i_{1}\left[\left(x_{\mathrm{D} i}-x_{\mathrm{D} j}\right) \sqrt{z}\right]\right\}=b(j, i) .
\end{aligned}
$$

(3) If $j=i$,

$$
\begin{aligned}
& \int_{x_{\mathrm{D} i-1}}^{x_{\mathrm{D} i}} K_{0}\left[\left(x_{\mathrm{D} j}-\sigma\right) \sqrt{z}\right] d \sigma \\
& =\int_{x_{\mathrm{D} i-1}}^{x_{\mathrm{D} j}} K_{0}\left[\left(x_{\mathrm{D} j}-\sigma\right) \sqrt{z}\right] d \sigma \\
& \quad+\int_{x_{\mathrm{D} j}}^{x_{\mathrm{D} i}} K_{0}\left[\left(\sigma-x_{\mathrm{D} j}\right) \sqrt{z}\right] d \sigma \\
& =\frac{1}{\sqrt{z}}\left\{\pi-2 K i_{1}\left[0.5 x_{\mathrm{D}} \sqrt{z}\right]\right\}=a(j, i), \\
& \int_{x_{\mathrm{D} i-1}}^{x_{\mathrm{D} i}} I_{0}\left[\left(x_{\mathrm{D} j}-\sigma\right) \sqrt{z}\right] d \sigma \\
& =\int_{x_{\mathrm{D} i-1}}^{x_{\mathrm{D} j}} I_{0}\left[\left(x_{\mathrm{D} j}-\sigma\right) \sqrt{z}\right] d \sigma \\
& \quad+\int_{x_{\mathrm{D} j}}^{x_{\mathrm{D} i}} I_{0}\left[\left(\sigma-x_{\mathrm{D} j}\right) \sqrt{z}\right] d \sigma \\
& =\frac{2}{\sqrt{z}}\left\{I i_{1}\left[0.5 x_{\mathrm{D}} \sqrt{z}\right]\right\}=b(j, i) .
\end{aligned}
$$

(4)

$$
\begin{aligned}
& \int_{x_{\mathrm{D} i-1}}^{x_{\mathrm{D} i}} K_{0}\left[\left(x_{\mathrm{D} j}+\sigma\right) \sqrt{z}\right] d \sigma \\
& =\frac{1}{\sqrt{z}}\left\{K i_{1}\left[\left(x_{\mathrm{D} j}+x_{\mathrm{D} i-1}\right) \sqrt{z}\right]\right. \\
& \left.-K i_{1}\left[\left(x_{\mathrm{D} j}+x_{\mathrm{D} i}\right) \sqrt{z}\right]\right\}=c(j, i),
\end{aligned}
$$

$$
\begin{aligned}
& \int_{x_{\mathrm{D} i-1}}^{x_{\mathrm{D} i}} I_{0}\left[\left(x_{\mathrm{D} j}+\sigma\right) \sqrt{z}\right] d \sigma \\
& \quad=\frac{1}{\sqrt{z}}\left\{I i_{1}\left[\left(x_{\mathrm{D} j}+x_{\mathrm{D} i-1}\right) \sqrt{z}\right]\right. \\
& \left.\quad-I i_{1}\left[\left(x_{\mathrm{D} j}+x_{\mathrm{D} i}\right) \sqrt{z}\right]\right\}=d(j, i) .
\end{aligned}
$$

And the second-order integral of (19) can be expressed as

$$
\begin{aligned}
\int_{0}^{x_{\mathrm{D}}} & \int_{0}^{v} \tilde{q}_{\mathrm{fD}}(u) d u d v \\
= & x_{\mathrm{D} j} \int_{0}^{x_{\mathrm{D} j}} \tilde{q}_{\mathrm{fD}}(u, s) d u-\int_{0}^{x_{\mathrm{D} j}} \tilde{q}_{\mathrm{fD}}(v, s) v d v \\
= & \sum_{i=1}^{j-1} \widetilde{q}_{\mathrm{fD} j}\left[\frac{\Delta x_{\mathrm{D}}^{2}}{2}+\left(x_{\mathrm{D} j}-i \Delta x_{\mathrm{D}}\right) \Delta x_{\mathrm{D}}\right] \\
& +\frac{\widetilde{q}_{\mathrm{fD} j}(s)}{8} \Delta x_{\mathrm{D}}^{2} .
\end{aligned}
$$

\section{Assume}

$$
\begin{aligned}
E(j, i)= & \frac{1}{2}[a(j, i)+c(j, i)] \\
& +\frac{1}{2} \frac{K_{1}\left(r_{\mathrm{eD}} \sqrt{z}\right)}{I_{1}\left(r_{\mathrm{eD}} \sqrt{z}\right)}[b(j, i)+d(j, i)], \\
F(j, i)= & \int_{0}^{x_{\mathrm{D} j}} \int_{0}^{v} \widetilde{q}_{\mathrm{fD}}(u) d u d v \\
= & \sum_{i=1}^{j-1} \tilde{q}_{\mathrm{fD} j}\left[\frac{\Delta x_{\mathrm{D}}^{2}}{2}+\left(x_{\mathrm{D} j}-i \Delta x_{\mathrm{D}}\right) \Delta x_{\mathrm{D}}\right] \\
& +\frac{\tilde{q}_{\mathrm{fD} j}(s)}{8} \Delta x_{\mathrm{D}}^{2} .
\end{aligned}
$$

And then

$j=1$ :

$$
\begin{gathered}
\widetilde{p}_{\mathrm{wD}}(s)+\left(\frac{\pi F(1,1)}{c_{\mathrm{fD}}}-\frac{E(1,1)}{2}\right) \widetilde{q}_{\mathrm{fD} 1}(s) \\
-\frac{1}{2} \sum_{i=1}^{n} \widetilde{q}_{\mathrm{fD} i}(s) E(1, i)=\frac{\pi x_{\mathrm{D} 1}}{s c_{\mathrm{fD}}},
\end{gathered}
$$

$j=2$ :

$$
\begin{gathered}
\tilde{p}_{\mathrm{wD}}(s)+\left(\frac{\pi F(2,1)}{c_{\mathrm{fD}}}-\frac{E(2,1)}{2}\right) \tilde{q}_{\mathrm{fD} 1}(s) \\
+\left(\frac{\pi F(2,2)}{c_{\mathrm{fD}}}-\frac{E(2,2)}{2}\right) \widetilde{q}_{\mathrm{fD} 2}(s) \\
-\frac{1}{2} \sum_{i=1}^{n} \widetilde{q}_{\mathrm{fD} i}(s) E(2, i)=\frac{\pi x_{\mathrm{D} 2}}{s c_{\mathrm{fD}}},
\end{gathered}
$$


$j=3$ :

$$
\begin{gathered}
\widetilde{p}_{\mathrm{wD}}(s)+\left(\frac{\pi F(3,1)}{c_{\mathrm{fD}}}-\frac{E(3,1)}{2}\right) \widetilde{q}_{\mathrm{fD} 1}(s) \\
+\left(\frac{\pi F(3,2)}{c_{\mathrm{fD}}}-\frac{E(3,2)}{2}\right) \widetilde{q}_{\mathrm{fD} 2}(s) \\
+\left(\frac{\pi F(3,3)}{c_{\mathrm{fD}}}-\frac{E(3,3)}{2}\right) \widetilde{q}_{\mathrm{fD} 3}(s) \\
-\frac{1}{2} \sum_{i=1}^{n} \widetilde{q}_{\mathrm{fD} i}(s) E(3, i)=\frac{\pi x_{\mathrm{D} 3}}{s c_{\mathrm{fD}}}
\end{gathered}
$$

$j=50$.

In addition to above expressions, due to steady flow, we also have

$$
\Delta x_{\mathrm{D}} \sum_{i=1}^{50} \widetilde{q}_{\mathrm{fD} i}(s)=\frac{1}{s} .
$$

Combining (30)-(33), we can get the linear equations as follows:

where $W(i, j)$ is coefficient of $\tilde{q}_{\mathrm{fD} k}(k=1,2, \ldots, 50)$.

The wellbore pressure of a hydraulic fractured well under constant production in naturally fractured reservoirs is obtained after solving (34) by using a Gaussian elimination approach.

In Laplace domain, we can easily consider the effect of skin factor

$$
\widetilde{p}_{s \mathrm{wD}}(s)=\frac{s \widetilde{p}_{\mathrm{wD}}(s)+S}{s}
$$

According to the Duhamel principle [23], the pressure solution under constant rate and the rate solution under constant pressure have the following correlation in Laplace domain:

$$
\widetilde{q}_{\mathrm{Dd}}(s)=\frac{1}{s^{2}} \widetilde{p}_{s \mathrm{wD}}(s)
$$

Combining (35) and (36), we can obtain the correlation between dimensionless rate $q_{\mathrm{D}}\left(t_{\mathrm{D}}\right)$ and dimensionless time $t_{\mathrm{D}}$ for any given parameters.

\section{Results and Discussion}

3.1. Validation of Solution. Riley [24] gave an analytical solution for elliptical finite conductivity fractures without volume fracturing. To validate the solution presented in this paper, we compared our solution with Riley's result. In our model, storage capacity ratio $\omega$, crossflow coefficient $\lambda$, and skin factor $S$ are considered to be equal to zero. Figure 2 shows the comparison of the two solutions under different fracture conductivity $C_{\mathrm{fD}}$. The good agreement validates the solution obtained in this work.

Combined with fracturing design, micro seismic detection and well test data, the parameters of a volume fractured well in tight oil reservoir are obtained, shown in Table 1. In Figure 3 it shows that the fractured well has produced for more than 200 days. After a short increasing period and stable period, the production began to decline. The calculation of established model is used to fit the actual production data at the decline stage. The calculated results are in good agreement with the actual production.

3.2. Typical Curves Analysis. The pressure and its derivative curves are presented in Figure 4, which shows basic flow characteristics for a volume fractured well in tight oil reservoir under different parameters by using Stehfest numerical inversion. The parameters are given as $C_{\mathrm{fD}}=0.1, \omega=$ 0.00001 , and $\lambda=0.01 ; C_{\mathrm{fD}}=1, \omega=0.01$, and $\lambda=0.01$; $C_{\mathrm{fD}}=10$ (infinite boundary), $\omega=0.005$, and $\lambda=0.0001$; $C_{\mathrm{fD}}=100, \omega=0.01$, and $\lambda=0.0001 ; C_{\mathrm{fD}}=300, \omega=0.01$, and $\lambda=0.0001$. As shown in Figure 4 , the flow can be divided into 7 stages:

(1) Stage A: Early Bilinear Flow (Artificial Fracture and Fractured Reservoirs Near the Wellbore). In this stage, the segment has a straight line with slope equal to $1 / 4$, demonstrating the bilinear flow region (see Figure 4). In this region, fluid flows through fracture to wellbore and from reservoirs to fracture at the same time. This region could be identified only if the fracture conductivity is relatively small.

(2) Stage B: Early Coupled Boundary Flow. In this stage, when the effect of volume fracturing is good, the artificial main fracture conductivity is much larger than that of the double medium fracture system. The fluid in the artificial main fracture reaches wellbore rapidly. However, the double medium fracture system 


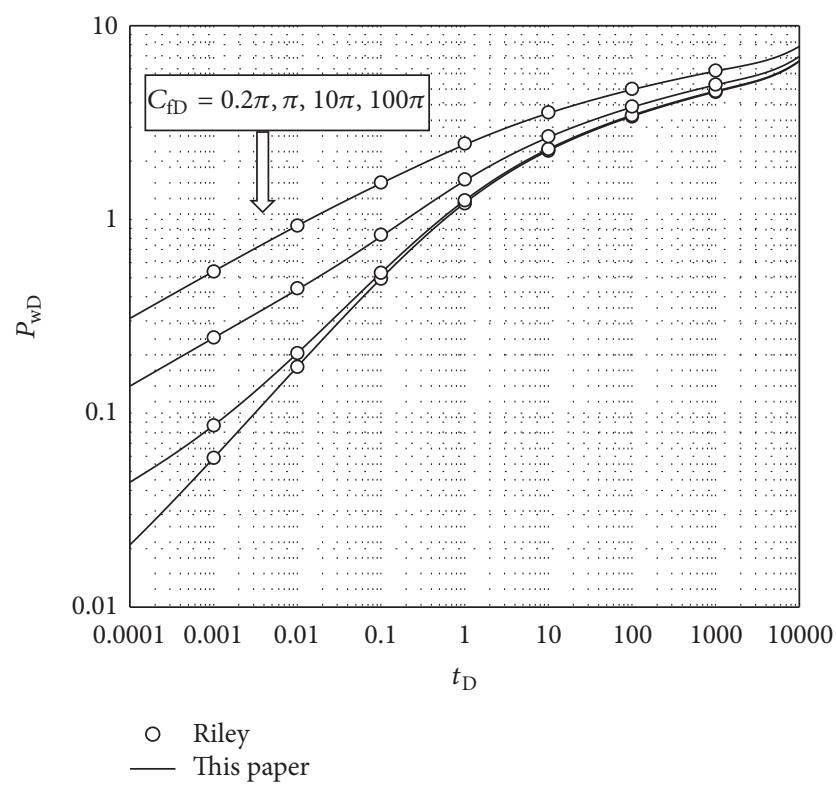

FIGURE 2: The comparison for the results of this paper and Riley [24].

TABLE 1: Parameters' value for influential factors analysis.

\begin{tabular}{lc}
\hline Parameter & Value \\
\hline Initial pressure $p_{e} / \mathrm{MPa}$ & 28.8 \\
Bottom pressure $p_{w} / \mathrm{MPa}$ & 8.8 \\
Initial oil viscosity $\mu_{o} / \mathrm{mPa} \cdot \mathrm{s}$ & 2.3 \\
Initial oil density $\rho_{o} / \mathrm{kg} \cdot \mathrm{m}^{-3}$ & 815 \\
Reservoir matrix permeability $k_{m} / \mu \mathrm{m}^{2}$ & 0.0001 \\
Formation thickness $h / \mathrm{m}$ & 12 \\
Wellbore radius $r_{w} / \mathrm{m}$ & 0.1 \\
Oil volume factor $B_{o}$ & 1.057 \\
Artificial main fracture half length $x_{f} / \mathrm{m}$ & 180 \\
Artificial main fracture permeability $k_{f} / \mu \mathrm{m}^{2}$ & 50 \\
Artificial main fracture width $w_{f} / \mathrm{mm}^{2}$ & 4 \\
Transformation radius $r_{\mathrm{eD}}$ & 15 \\
\hline
\end{tabular}

cannot provide adequate fluid supply. Both pressure and pressure derivate curves increase, similar to the transient pressure response of weak energy supply or closed boundary reservoirs.

(3) Stage C: Early Linear Flow (Fractured Reservoir Near the Wellbore). The pressure and pressure derivative curves are both straight lines with slope equal to $1 / 2$, which clearly demonstrates early linear flow. In this region, linear fluid flow from formation to the artificial fracture reduces the seepage resistance. The early $A$ and $B$ flow regions do not necessarily occur for each fracturing treatment, which depends on the conductivity of artificial fracture flow. As shown in Figure 4, the bilinear flow is more obvious in the case of smaller fracture conductivity.

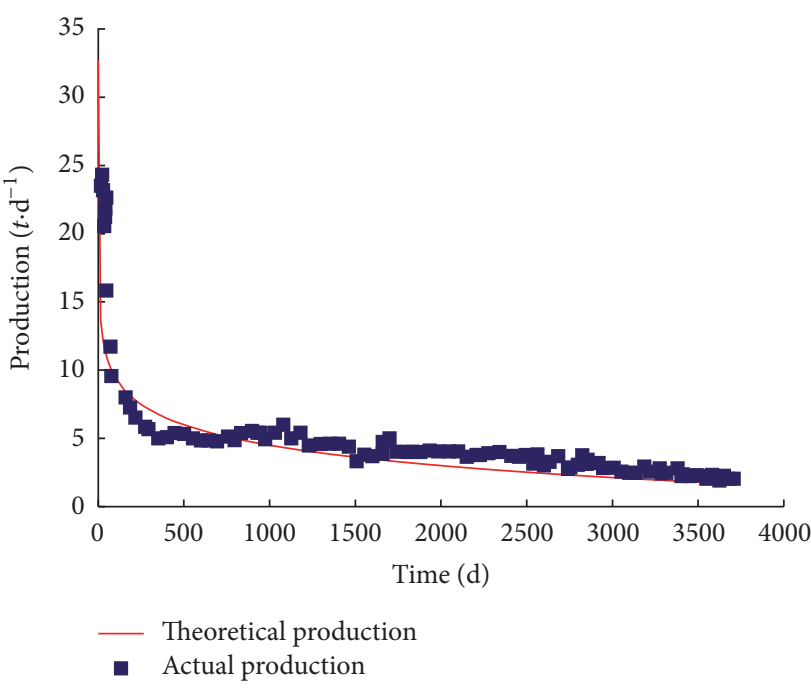

Figure 3: Actual and theoretical production data fitted curves.

(4) Stage D: Mid Radial Flow (Microcracks in the Fractured Formation). The segment has a straight line with 0.5 constant.

(5) Stage E: Mid Bilinear Flow or Pseudo Steady-State Flow. The larger the crossflow factor $\lambda$ is, the earlier the crossflow happens. Before fluid crossflow between microcracks and formation occurs, it exhibits pseudo steady flow or mid linear flow briefly which is affected by the crossflow coefficient $\lambda$. Specifically, if $\lambda$ is small it will exhibit pseudo steady flow when the pressure reaches the boundary or linear flow when the pressure wave disturbance does not reach the boundary.

(6) Stage F: Mid Crossflow (Matrix and Fracture). Due to the low permeability of matrix and the slow pressure drop, crossflow will occur between the matrix and fracture. And the pressure derivative curve is concave. Compared to the conventional dual media the crossflow will happen earlier. At the same time, due to the dimensionless setting, the crossflow coefficient $\lambda$ is 2 to 4 orders of magnitude larger than that of the conventional dual medium.

(7) Stage G: Late Pseudo Steady Flow. For infinite outer boundary, the pressure derivative curve is a horizontal line. However, for closed outer boundary, the slopes of pressure and pressure derivative cures are 1. In some cases, affected by the storage capacity ratio $\omega$ and crossflow coefficient $\lambda$ the medium segment has different flow characteristics: D + E or D + F.

3.3. Blasingame Type Curves and Discussions. The general definitions of the base decline for type curve variables can be given by

$$
\begin{aligned}
& q_{\mathrm{Dd}}=q_{\mathrm{D}} b_{\mathrm{Dpss}}, \\
& t_{\mathrm{Dd}}=\frac{2}{b_{\mathrm{Dpss}}\left(r_{\mathrm{eD}}^{2}-1\right)} t_{D} .
\end{aligned}
$$




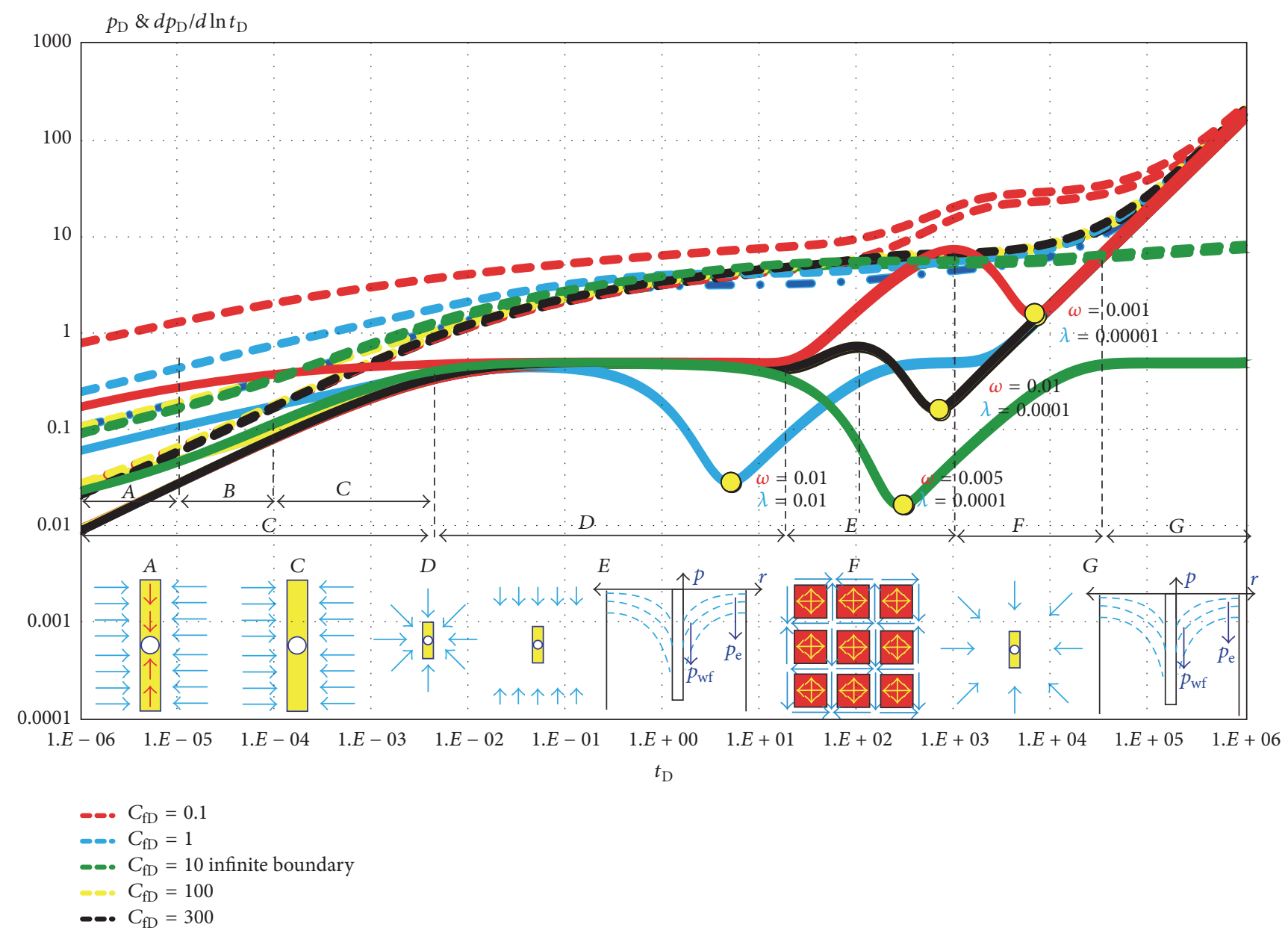

FIgURE 4: Flow stage division.

The definitions of (37) were presented by Fetkovich [26]. However, to introduce material balance time, we use (35) as a constant rate solution [27] and then use $1 / p_{\mathrm{wD}}$ result as $q_{\mathrm{D}}$. To eliminate multiple solutions and errors, integral average method of rate was created by Blasingame et al. [28]. The auxiliary variables in this method typically used for decline type curve analysis are given by

(1) rate integral function: $q_{\mathrm{Ddi}}$

$$
q_{\mathrm{Ddi}}=\frac{N_{p \mathrm{Dd}}}{t_{\mathrm{Dd}}}=\frac{1}{t_{\mathrm{Dd}}} \int_{0}^{t_{\mathrm{Dd}}} q_{\mathrm{Dd}}(\tau) d \tau,
$$

(2) rate integral derivative function: $q_{\text {Ddid }}$

$$
q_{\mathrm{Ddid}}=-\frac{d q_{\mathrm{Ddi}}}{d \ln \left(t_{\mathrm{Dd}}\right)}=-t_{\mathrm{Dd}} \frac{d q_{\mathrm{Ddi}}}{d t_{\mathrm{Dd}}}=q_{\mathrm{Ddi}}-q_{\mathrm{Dd}} .
$$

Now incorporating (37)-(39) with the flow model assumed previously, we can consequently establish new Blasingame type curves for volume fractured vertical wells in fractured tight oil reservoirs, which are shown in Figures 5-17.
3.3.1. Comparison between Volume Fracturing and Conventional Fracturing. The comparison of vertical well productivity with the same main fracture length between conventional fracturing and volume fracturing is shown in Figures 5 and 6. From Figure 5 we can see that the productivity of volume fracturing is obviously higher than that of conventional fracturing and it has nonlinear characteristics. Due to tightness of formation, complexity of pore structure, and low permeability of matrix, the vertical well productivity is still very low after conventional fracturing. However, volume fracturing can form a fracture network in formation which greatly improves the permeability of the whole reservoir, reduces the seepage resistance from the matrix to the main fracture, increases the contact area between main fracture and formation matrix, and shortens the flow distance from the matrix to the main fracture. Therefore, in order to get higher productivity and achieve economic demand, volume fracturing must be carried out.

3.3.2. Storage Capacity Ratio $w$ and Crossflow Coefficient $\lambda$. The effects of storage capacity ratios $(\omega=0.01,0.05,0.1$, $0.5,1)$ and crossflow coefficients $(\lambda=0.0001,0.001,0.01)$ on the rate decline curves are given in Figures 7 and 8, 


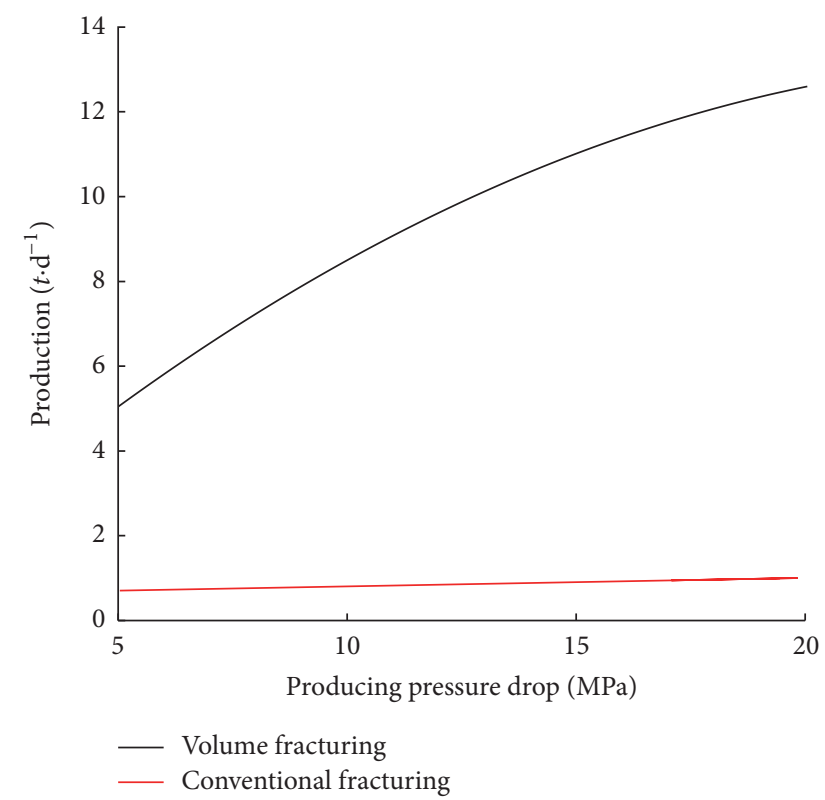

Figure 5: Comparison of vertical well productivity between conventional fracturing and volume fracturing under the same pressure drop.

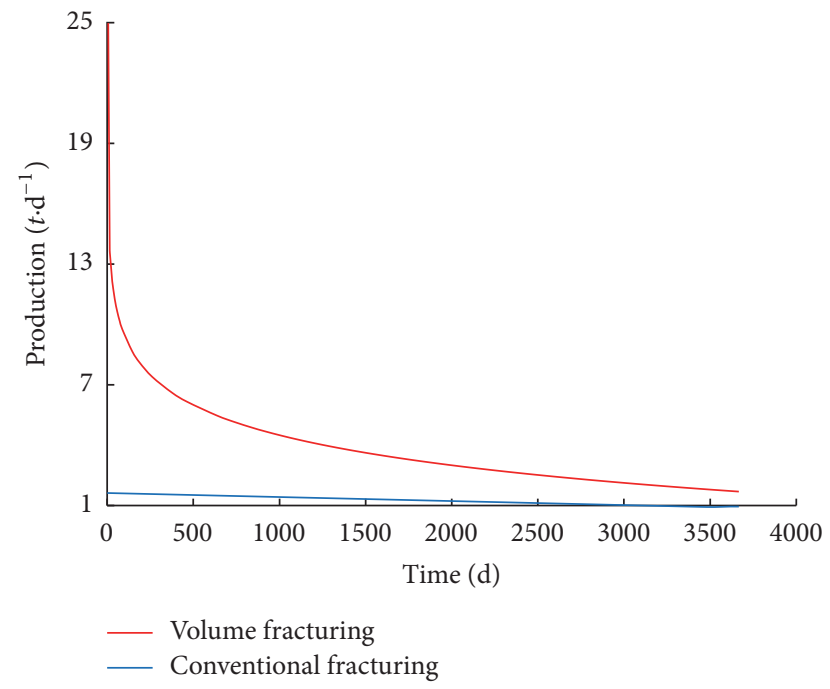

Figure 6: Comparison of vertical well productivity between conventional fracturing and volume fracturing at different time.

respectively. From these two figures, we can see that storage capacity ratio and crossflow coefficient affect the time and degree of crossflow, respectively, similar to the common dual media. The storage capacity ratio has an influence on the production of transitional flow. The smaller the storage capacity ratio $\omega$ is, the more obvious the crossflow is. At the intermediate time, the curve of production is sunken. The values of both $q_{\text {Ddi }}$ and $q_{\text {Ddid }}$ decrease with the increase of storage capacity ratio $\omega$. In pseudo steady-state flow, curves of both groups normalize, respectively. The larger the storage capacity ratio $\omega$, the smaller the peak value of production. The

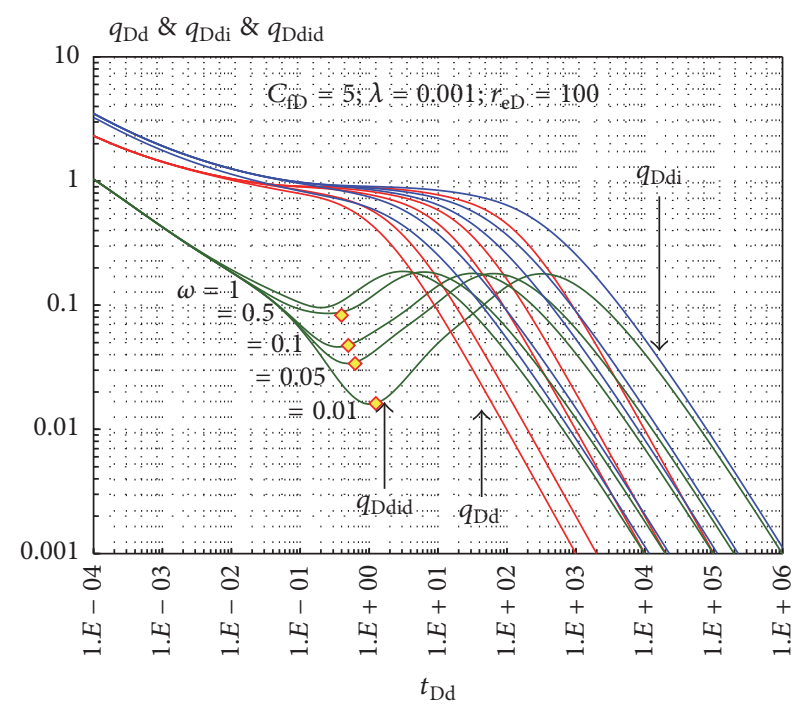

FIGURE 7: The effect of storage capacity ratio factor on type curves.

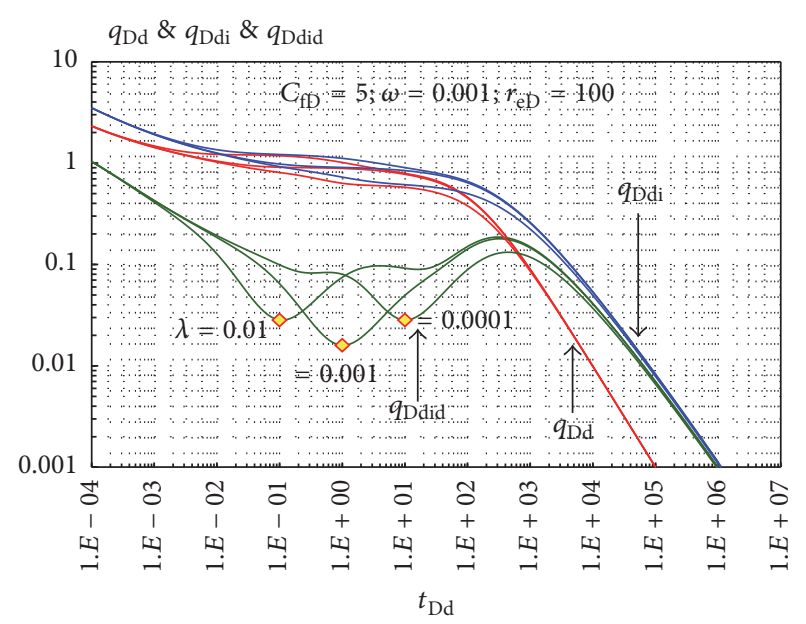

Figure 8: The effect of crossflow coefficient factor on type curves.

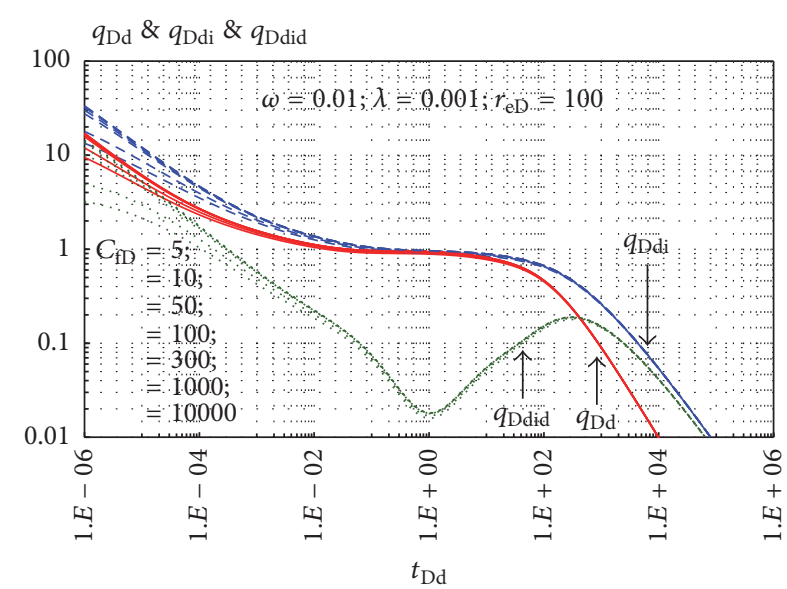

Figure 9: The effect of fracture conductivity on type curves.

crossflow coefficient $\lambda$ ratio has an influence on the production of matrix-fracture crossflow. The greater the crossflow 


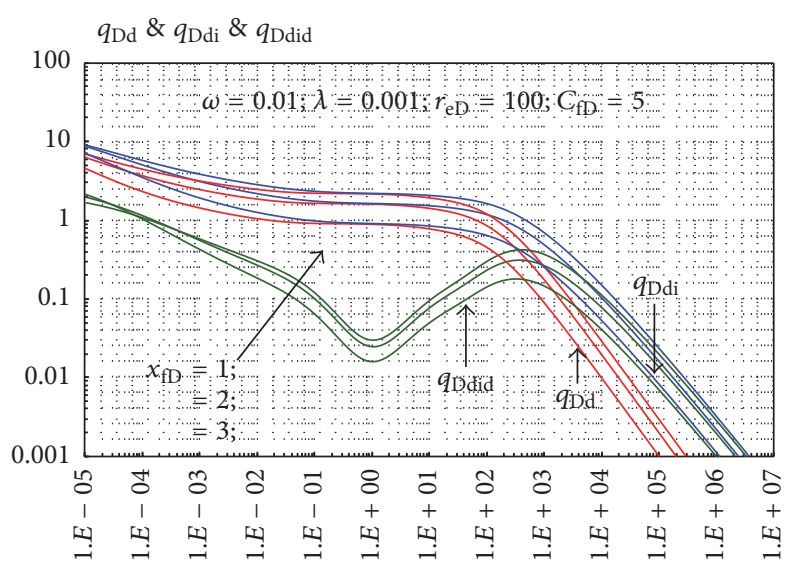

Figure 10: The effect of fracture length on type curves.

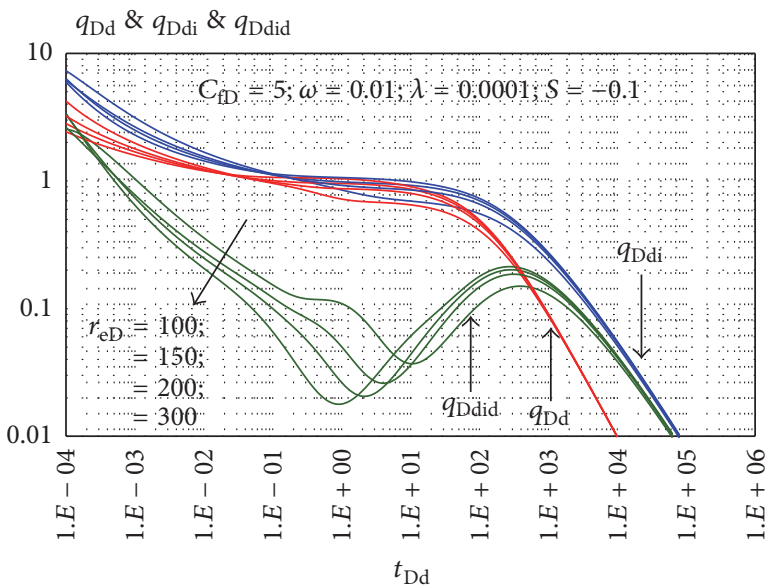

FIGURE 11: The effect of drainage radius for volume fracturing on type curves.

coefficient $\lambda$ is, the earlier the crossflow happens. Figure 8 shows that if the crossflow coefficient $\lambda$ is too small, when the pressure wave does not touch the boundary, the medium linear flow occurs and after the pressure wave reaches the boundary, pseudo steady flow will occur. Similarly, under the condition of same storage capacity ratio $\omega$, the values of both $q_{\text {Ddi }}$ and $q_{\text {Ddid }}$ increase with the increase of crossflow coefficient $\lambda$ in transient flow and normalize, respectively, in pseudo steady-state flow.

\subsubsection{Fracture Conductivity $c_{f D}$ and Fracture Half Length} $x_{f D}$. The effect of artificial fracturing is to leave a high permeability channel near the well formation, which is convenient for fluid to flow from the far well zone to the bottom hole. Figures 9 and 10 show the effect of fracture conductivity $C_{\mathrm{fD}}\left(C_{\mathrm{fD}}=5,10,50,100,300,1000,10000\right)$ and length $x_{\mathrm{fD}}\left(x_{\mathrm{fD}}=1,2,3\right)$ on dimensionless rate integral $q_{\text {Ddi }}$ and rate integral derivative $q_{\text {Ddid }}$ for the same $\omega, \lambda, r_{\mathrm{eD}}$, and $S$. From Figure 9, we find that the effect of $C_{\mathrm{fD}}$ on $q_{\mathrm{Ddi}}$ and $q_{\text {Ddid }}$ is only in the early stage. The values of both $q_{\text {Ddi }}$ and $q_{\text {Ddid }}$ increase with the increase of fracture conductivity. However, to a certain extent, the effect of fracture length is

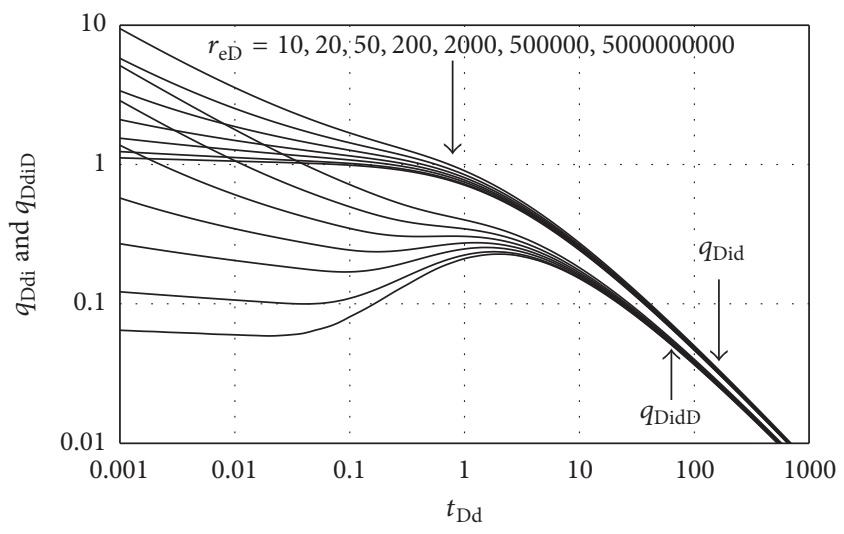

FIgURE 12: The effect of drainage radius for conventional fracturing on type curves [25].

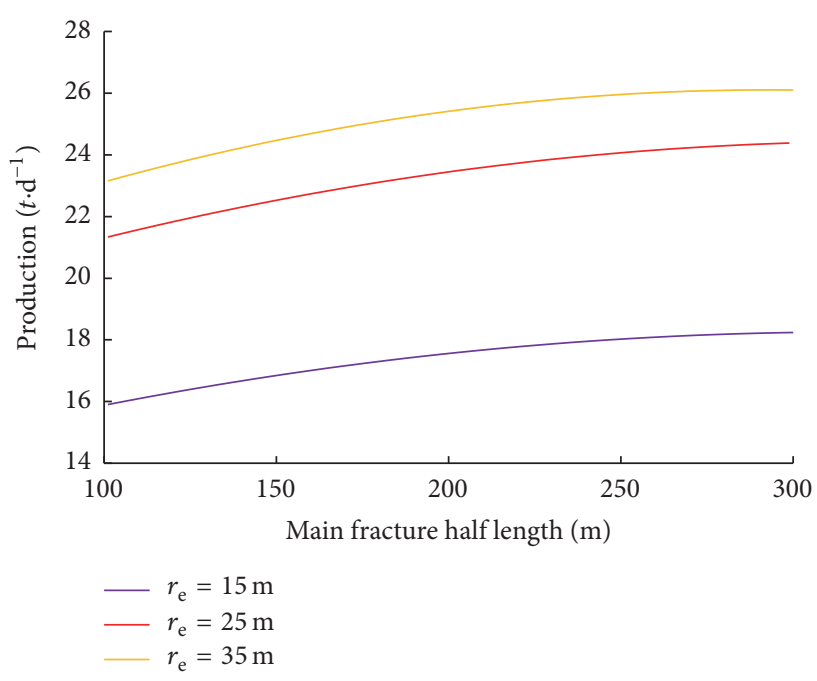

FIGURE 13: Effect of stimulated reservoir volume on vertical well productivity.

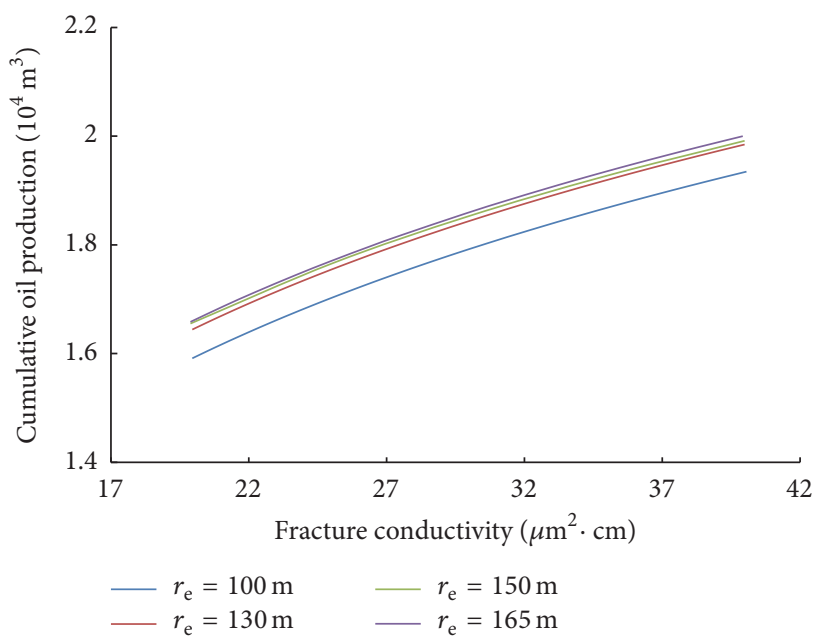

FIGURE 14: Effect of fracture conductivity on cumulative oil production. 


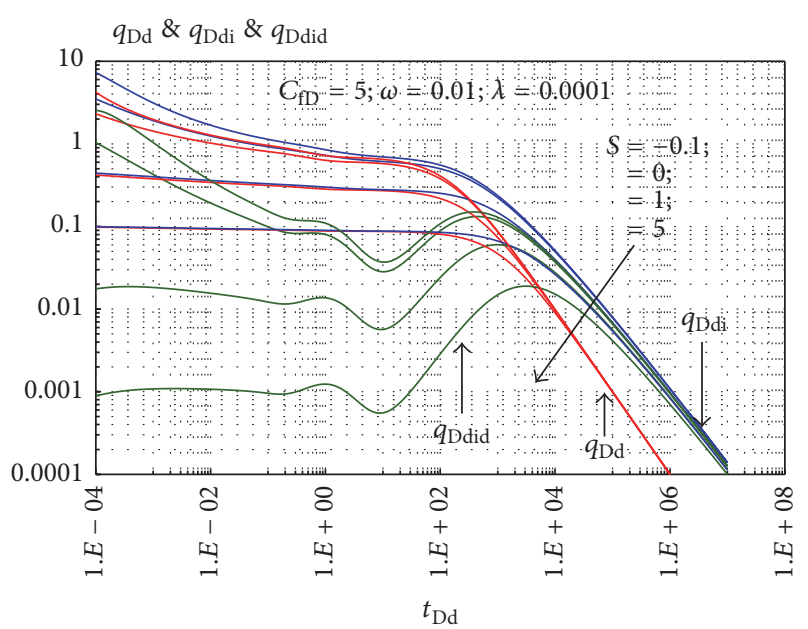

FIGURE 15: The effect of skin factor on type curves.

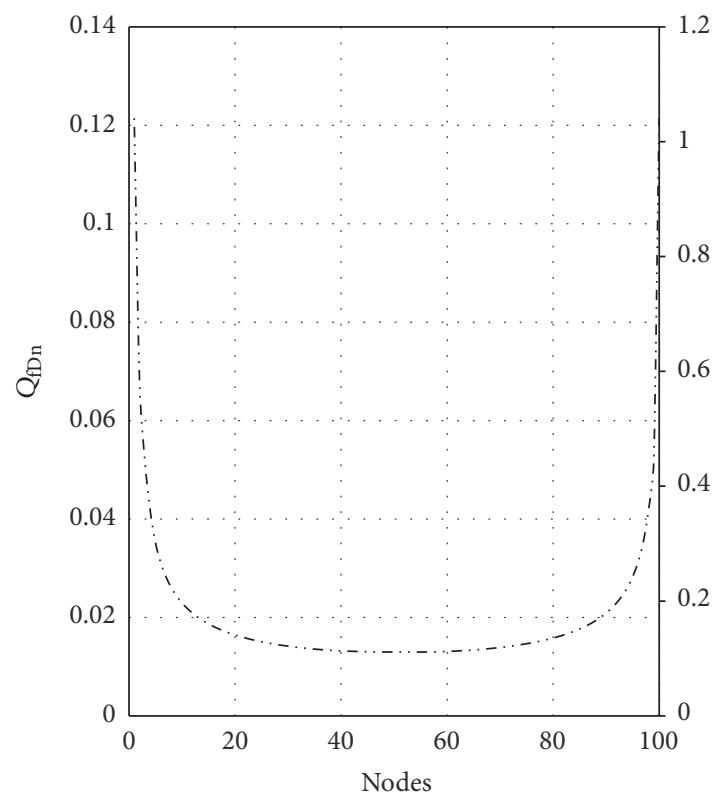

FIGURE 16: Flow distribution along fracture surface.

more obvious than that of fracture conductivity on improving production effect. By combining Figures 9 and 10, we can see that, with the increase of fracture conductivity, the increase rate of production gradually reduces. When the fracture conductivity $C_{\mathrm{fD}}$ is more than 300 , the effect of $C_{\mathrm{fD}}$ on $q_{\mathrm{Ddi}}$ and $q_{\text {Ddid }}$ is little and can be ignored. However, with the increase of fracture length, the increase rate of production is basically changeless. The longer the fracture length is, the higher the production and the longer the stable period will be. The essence of fracture conductivity is the amount of fluid from reservoir to fracture per unit pressure gradient. In tight oil reservoirs, the fluid supply of the reservoir matrix to the main fracture is limited; that is, the conductivity is low. As the length of fracture increases, more fracture network formed by the volume fracturing is effectively connected with the main fracture, forming a larger supply area and improving

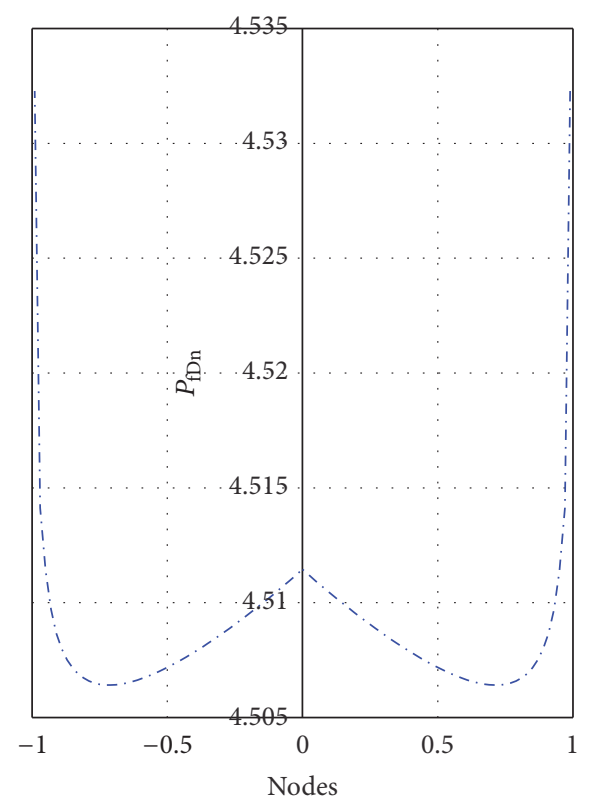

FIgURE 17: Pressure distribution along fracture surface.

the overall yield. In conventional fractured reservoirs, the main fracture conductivity is dominant, whereas in volume fractured tight oil reservoirs the effect of fracture length on productivity is dominant which is contrary to normal expectation. Therefore, in order to achieve the desired effect of volume fracturing design, a certain length of the main fracture should also be ensured.

3.3.4. Stimulated Reservoir Volume Area. Figure 11 shows the effect of drainage radius $r_{\mathrm{eD}}\left(r_{\mathrm{eD}}=100,150,200,300\right)$ on dimensionless production, rate integral $q_{\text {Ddi }}$, and rate integral derivative $q_{\mathrm{Ddid}}$ for the same $C_{\mathrm{fD}}, \omega, \lambda$, and $S$, respectively. It can be seen from Figure 11 that when dimensionless decline time is relatively smaller $\left(t_{\mathrm{dD}}<0.1\right)$, the production increases with the decrease of $r_{\mathrm{eD}}$, which is contrary to the conventional fracturing [28] (Figure 12). This is because the early flow mainly occurs between the main fracture and the microcracks. In the case of the same length of the main fracture, the smaller the drainage radius is, the higher the fracture penetration ratio and the higher the production in the early and middle period will be. When the values of time become larger $\left(t_{\mathrm{dD}}>0.1\right)$, the flow is mainly between the reservoir matrix and microcracks, and the smaller the volume of fracture network, the lower the fluid supply capacity, and the faster the production decline. $r_{\mathrm{eD}}$ can also affect the time and degree of crossflow. The greater the $r_{\mathrm{eD}}$ is, the earlier the crossflow happens, and the more obvious the crossflow is.

From Figure 13 we can see that, with the drainage radius of stimulated reservoir volume and length of main fracture increasing, the area of stimulated volume, well-controlled drainage area, and well productivity also increase, but the increase rate reduces.

From Figure 14 we can see that when the stimulated reservoir volume is constant, the greater the fracture conductivity and the higher the cumulative oil production. 
TABLE 2: Basic parameters of fracture and formation.

\begin{tabular}{lc}
\hline Parameter & Value \\
\hline Elastic storativity ratio $\omega$ & 0.01 \\
Crossflow coefficient $\lambda$ & 0.0001 \\
Formation permeability $k / \mathrm{mD}$ & 0.05 \\
Drainage radius $r_{e} / \mathrm{m}$ & 1000 \\
Half length of fracture $x_{f} / \mathrm{m}$ & 100 \\
Artificial fracture permeability $k_{f} / \mathrm{mD}$ & 50000 \\
Width of fracture $w_{f} / \mathrm{m}$ & 0.0023 \\
Dimensionless drainage $r_{\mathrm{eD}}$ & 100 \\
Dimensionless fracture conductivity $c_{\mathrm{fD}}$ & 300 \\
\hline
\end{tabular}

When the length and conductivity of main fracture are constant, the contribution of stimulated reservoir volume to the cumulative oil production is not obvious. This is mainly because the single well control reserve is very limited, and there is no sense in increasing the stimulated reservoir volume without limitation. At the same time, increasing the volume of reservoir reconstruction will also increase the scale of fracturing and construction difficulty. Therefore, when the stimulated reservoir volume is constant, the length of fracture should be increased so as to improve the fracture penetration, conductivity, and well production.

3.3.5. Skin Factor $S$. Figure 15 shows the effect of skin factor $S(S=0.1,1,0,5)$ on dimensionless production, rate integral $q_{\mathrm{Ddi}}$, and rate integral derivative $q_{\mathrm{Ddid}}$ for the same $C_{\mathrm{fD}}, \omega$, $\lambda$, and $r_{\mathrm{eD}}$, respectively. From Figure 15 we can conclude that the production, rate integral $q_{\mathrm{Ddi}}$, and rate integral derivative $q_{\text {Ddid }}$ decrease with the increase of skin factor. When the volume fracturing effect is good, the value of skin factor will be small and even be negative. Skin factor does not affect the time and degree of crossflow.

3.3.6. Flow and Pressure Distribution of Fracture Surface. By using (34) we can obtain the flow and pressure distribution along the fracture surface. Basic parameters of the artificial main fracture and formation after volume fracturing are given in Table 2.

The flow and pressure distribution along the fracture surface are given in Figures 16 and 17. From Figures 16 and 17 we can see that the yield on both ends of the fracture is higher and the center of the fracture is lower. This is because the drainage area $r_{\mathrm{eD}}$ on both ends of the fracture is much larger than the center. The pressure distribution on the fracture is proportional to the flow distribution. The better the volume modification is, the longer the stable production period will be.

\section{Conclusions}

In this study, we have investigated the productivity characteristics of a volume fractured vertical well in tight oil reservoir. The specific conclusions are as follows:
(1) Using Laplace transform and Stehfest numerical inversion method, a semianalytical solution for the vertical well with reconstructed fractured network is established in fractured tight oil reservoirs. The effects of an artificial main fracture near wellbore were also taken into account in this model and it can simply reflect the flow characteristics of the production wells in each stage after fracturing and acidizing treatment. Combining the calculated results with actual production data shows a good fitting performance.

(2) Based on the established model, new type curves are established to analyze the flow characteristics, which can be divided into seven stages: (a) linear flow in artificial main fracture; (b) coupled boundary flow; (c) early linear flow in fractured formation; (d) mid radial flow in the microcracks of the formation; (e) mid radial flow or pseudo steady flow; (f) mid crossflow; (g) closed boundary flow.

(3) Effects of some sensitive parameters such as storage capacity ratio, crossflow coefficient, fracture conductivity, fracture length, and skin factor are also analyzed in detail. Storage capacity ratios and crossflow coefficients affect the time and degree of crossflow, respectively. Artificial fracturing can leave a high permeability channel in the near well formation, which is convenient for the fluid to flow from the well zone to the bottom hole. The production increases with the increase of artificial main fracture conductivity. To a certain extent, the effect of fracture length is more obvious than that of fracture conductivity on improving production effect. When the length and conductivity of the main fracture are constant, the contribution of stimulated reservoir volume to the cumulative oil production is not obvious. And there is no sense in increasing the stimulated reservoir volume without limitation. When the stimulated reservoir volume is constant, the length of fracture should also be increased so as to improve the fracture penetration and well production.

\section{Nomenclature}

Dimensionless Variables: Real Domain

$b_{\text {Dpss }}:$ Dimensionless pseudo steady-state constant

$C_{\mathrm{fD}}$ : Dimensionless artificial main fracture conductivity

$p_{\mathrm{wD}}$ : Dimensionless well bottom pressure

$p_{2 \mathrm{fD}}$ : Dimensionless micro fracture pressure in volume modification region

$p_{\mathrm{fD}}$ : Dimensionless artificial main fracture pressure

$q_{\text {Dd }}:$ Dimensionless decline rate

$q_{\text {Ddi }}$ : Dimensionless decline rate integral

$q_{\text {Ddid }}:$ Dimensionless decline rate integral derivative 
$t_{\mathrm{D}}:$ Dimensionless time

$t_{\mathrm{Dd}}$ : Dimensionless decline time

$x_{\mathrm{D} i}$ : Midpoint of the $i$ segment

$\gamma$ : Euler constant, 05771.

\section{Dimensionless Variables: Laplace Domain}

$\widetilde{p}_{\mathrm{D}}$ : The pressure $p_{\mathrm{D}}$ in Laplace domain

$\widetilde{p}_{\mathrm{wD}}$ : The pressure $p_{\mathrm{wD}}$ in Laplace domain

$\widetilde{p}_{2 \mathrm{fD}}$ : Dimensionless micro fracture pressure $p_{2 \mathrm{fD}}$ in Laplace domain

$\widetilde{p}_{\mathrm{fD}}$ : Artificial main fracture pressure $p_{\mathrm{fD}}$ in volume modification region in Laplace domain

$\widetilde{q}_{\text {Dd }}$ : Artificial main fracture pressure $q_{\text {Dd }}$ in Laplace domain

$\tilde{q}_{\mathrm{fD}}$ : The fracture rate $q_{\mathrm{fD}}$ in Laplace domain

$s:$ Time variable in Laplace domain, dimensionless.

\section{Field Variables}

A: Reservoir drainage area, $\mathrm{m}^{2}$

$c_{2 \mathrm{~m}}$ : Compressibility for matrix, $1 / \mathrm{Mpa}$

$c_{2 \mathrm{f}}$ : Compressibility for micro fracture, $1 / \mathrm{Mpa}$

$\phi_{2 \mathrm{~m}}$ : Porosity for matrix, fraction

$\phi_{2 \mathrm{f}}$ : Porosity for micro fracture

$k_{\mathrm{f}}$ : Permeability of artificial main fracture, $\mathrm{mD}$

$k_{2 \mathrm{f}}$ : Permeability of micro fracture, $\mathrm{mD}$

$k_{2 \mathrm{~m}}$ : Permeability of matrix, $\mathrm{mD}$

$p: \quad$ Formation pressure, Mpa

$p_{i}$ : Initial formation pressure, $\mathrm{Mpa}$

$r$ : Reservoir radius, $\mathrm{m}$

$r_{\mathrm{e}}: \quad$ Equivalent drainage radius, $\mathrm{m}$

$t$ : Time variable, days

$x_{\mathrm{f}}$ : Fracture half length, $\mathrm{m}$

$w$ : Fracture width, $\mathrm{m}$

$\omega_{2}$ : Elastic storativity ratio, fraction

$\lambda_{2}$ : Crossflow coefficient, fraction.

\section{Special Functions}

$K_{0}(x)$ : Modified Bessel function (2nd kind, zero order)

$K_{1}(x)$ : Modified Bessel function (2nd kind, first order)

$I_{0}(x)$ : Modified Bessel function (1st kind, zero order)

$I_{1}(x)$ : Modified Bessel function (1st kind, first order).

\section{Special Subscripts}

Dd: Dimensionless decline variable

i: Integral function (or initial value)

id: Integral derivative function

pss: Pseudo steady-state.

\section{Competing Interests}

The authors declare that there is no conflict of interests regarding the publication of this paper.

\section{Acknowledgments}

This paper was supported by the Fundamental Research Funds for the Central Universities (Grant no. 53200859545), the Ministry of Land and Resources Special Geological Survey: Upper Paleozoic Marine Shale Gas Geological Survey in Yunnan, Guizhou, Guangxi Region (Grant no. DD20160178), and the Key Laboratory of Unconventional Petroleum Geology of Geological Survey of China Open Fund and Major National R\&D Projects: Study on the Test Method for Shale Structure and Composition at Different Scales (Grant no. 2016ZX05034-003-006).

\section{References}

[1] C. Jia, C. Zou, J. Li, D. Li, and M. Zheng, "Assessment criteria, main types, basic features and resource prospects of the tight oil in China," Acta Petrolei Sinica, vol. 33, no. 3, pp. 343-350, 2012.

[2] C. Zou, R. Zhu, S. Wu et al., "Types, characteristics, genesis and prospects of conventional and unconventional hydrocarbon accumulations: taking tight oil and tight gas in China as an instance," Acta Petrolei Sinica, vol. 33, no. 2, pp. 173-187, 2012.

[3] J. Nan, S. Wang, W. Yao et al., "Micro-fractures in extra-low permeability reservoir of Yanchang Formation in Ordos Basin," Lithologic Reservoir, vol. 19, no. 4, pp. 40-44, 2007.

[4] Y. Guo, J. Liu, H. Yang et al., "Hydrocarbon accumulation mechanism of low permeable tight lithologic oil reservoirs in the Yanchang Formation, Ordos Basin, China," Petroleum Exploration and Development, vol. 39, no. 4, pp. 417-425, 2012.

[5] K. M. Al-Salem, M. A. Ali, and C. Lin, "Tight oil reservoir development feasibility study using finite difference simulation and streamlines," in Proceedings of the SPE Saudi Arabia Section Technical Symposium, Al-Khobar, Saudi Arabia, May 2009.

[6] C. L. Cipolla, C. Carbo, E. P. Lolon et al., "Reservoir modeling and production evaluation in shale-gas reservoirs," in Proceedings of the International Petroleum Technology Conference, Paper IPTC 13185, Doha, Qatar, 2009.

[7] R. Barry, "Accurate simulation of non-darcy flow in stimulated fractured shale reservoirs," in Proceedings of the SPE Western Regional Meeting, Paper SPE 132093, Anaheim, Calif, USA, May 2010.

[8] W. Wang, G. Zhao, Y. Su et al., "Application of network fracturing technology to tight oil reservoir," Xinjiang Petroleum Geology, vol. 34, no. 3, pp. 345-348, 2013.

[9] W.-D. Wang, Y.-L. Su, L.-J. Mu, M.-R. Tang, and L. Gao, "Influencing factors of stimulated reservoir volume of vertical wells in tight oil reservoirs," Journal of China University of Petroleum, vol. 37, no. 3, pp. 93-97, 2013.

[10] H. Arvind, D. Franz, C. Martin et al., "Volumetric Fracture Modeling Approach (VFMA): incorporating microseismic data in the simulation of shale gas reservoirs," in Proceedings of the SPE Annual Technical Conference and Exhibition, Paper SPE 134683, Florence, Italy, September 2010.

[11] C. M. Du, X. Zhang, L. Zhan et al., "Modeling hydraulic fracturing induced fracture networks in shale gas reservoirs as 
a dual porosity system," in Proceedings of the International Oil and Gas Conference and Exhibition in China, Beijing, China, June 2010.

[12] X. Liu and G. Zhao, "A fractal wormhole model for cold heavy oil production," Journal of Canadian Petroleum Technology, vol. 44, no. 9, pp. 31-36, 2005.

[13] X. Liu, G. Zhao, and Y. C. Jin, "Coupled reservoir/wormholes model for cold heavy oil production wells," Journal of Petroleum Science and Engineering, vol. 50, no. 3-4, pp. 258-268, 2006.

[14] X. Lei and Z. Gang, "A novel approach for determining wormhole coverage in CHOPS wells," in Proceedings of the SPE Heavy Oil Conference, Paper SPE 157935, Calgary, Canada, June 2012.

[15] X. Liu, The Research of Deliverability Evaluated for Volume Reconstruction in Tight oil Reservoir, China University of Geosciences, Beijing, China, 2013.

[16] X. Liu, C. B. Tian, and L. Y. Jiang, "Steady state deliverability evaluation model of fracture network reconstruction vertical well in tight oil reservoir," Journal of Northeast Petroleum University, vol. 38, no. 1, pp. 91-97, 2014.

[17] X. Liao and X. Chen, "Pressure transient analysis of volume fracturing well in low permeability oil reservoir," Science \& Technology Review, vol. 34, no. 7, pp. 117-122, 2016.

[18] H. Stehfest, "Algorithm 368: numerical inversion of Laplace transforms [D5]," Communications of the ACM, vol. 13, no. 1, pp. 47-49, 1970.

[19] J. E. Warren and P. J. Root, "The behavior of naturally fractured reservoirs," Society of Petroleum Engineers Journal, vol. 3, no. 3, 1963.

[20] J. Ge, Z. Ning, Y. Liu et al., Modern Reservoir Seepage Rule, Petroleum Industry Press, 2003.

[21] F. Medeiros, B. Kurtoglu, E. Ozkan et al., "Pressure-transient performance of hydraulically horizontal well in locally and globally naturally fractured formations," in Proceedings of the International Petroleum Technology Conference, Paper IPTC 11781-MS, Dubai, UAE, December 2007.

[22] L. Cinco, V. Samaniego, and A. Dominguez, "Transient pressure behavior for a well with a finite-conductivity vertical fracture," Society of Petroleum Engineers Journal, vol. 18, no. 4, pp. 253264, 1978.

[23] X. D. Wang, Mechanic Basis of Fluids Flowing in Porous Media, Petroleum Industry, Beijing, China, 2006.

[24] M. F. Riley, "Analytical solutions for elliptical finite-conductivity fractures," in Proceedings of the 66th Annual Technical Conference and E14, Paper SPE 22656, pp. 31-36, Dallas, Tex, USA, 1991.

[25] L. Wang, X.-D. Wang, X.-M. Ding, L. Zhang, and C. Li, "Rate decline curves analysis of a vertical fractured well with fracture face damage," Journal of Energy Resources Technology, vol. 134, no. 3, Article ID 032803, 9 pages, 2012.

[26] M. Fetkovich, "Decline curve analysis using type curves," Journal of Petroleum Technology, vol. 32, no. 6, pp. 1065-1077, 2013.

[27] T. A. Blasingame, J. L. Johnston, and W. J. Lee, "Type-curve analysis using the pressure integral method," in Proceedings of the SPE California Regional Meeting, Bakersfield, Calif, USA, April 1989.

[28] T. A. Blasingame, T. L. McCray, and W. J. Lee, "Decline curve analysis for variable pressure drop/variable flowrate systems," in Proceedings of the SPE Gas Technology Symposium, Paper no. SPE 21513, Houston, Tex, USA, January 1991. 


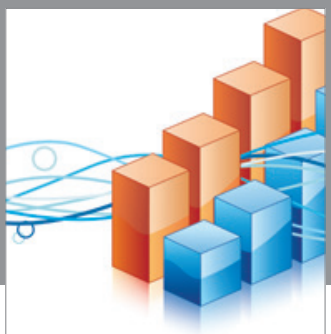

Advances in

Operations Research

vatem alat4

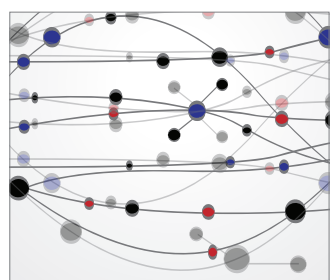

\section{The Scientific} World Journal
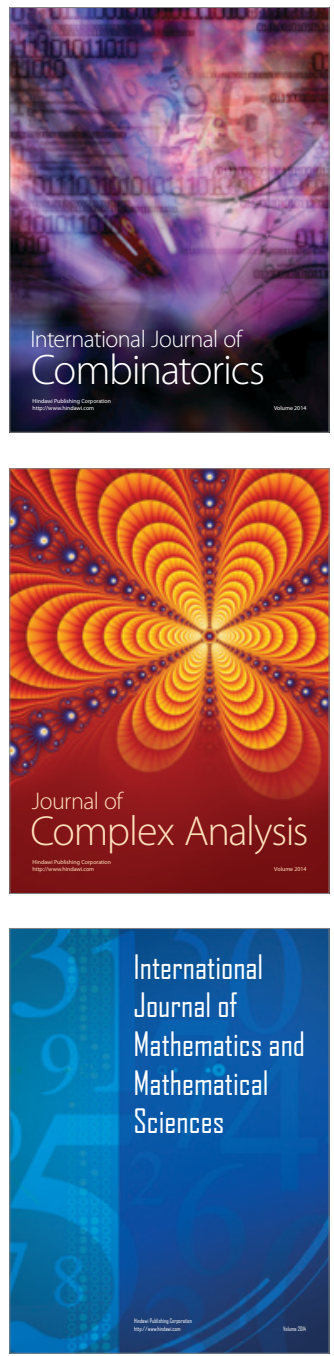
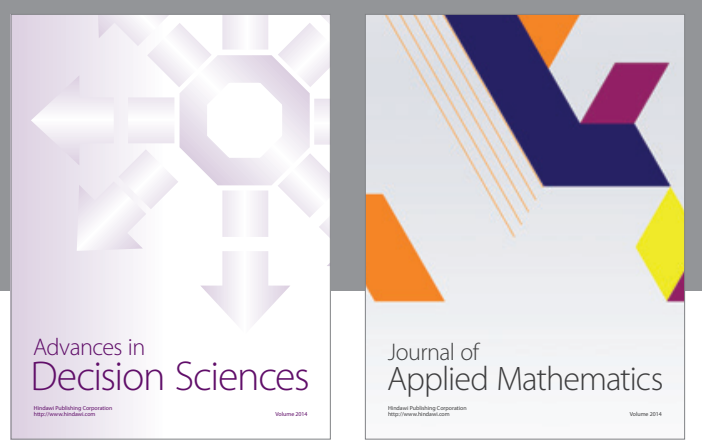

Algebra

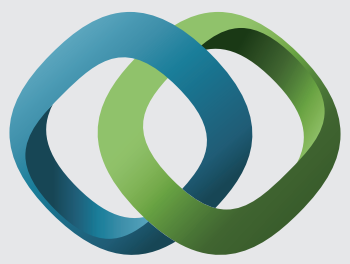

\section{Hindawi}

Submit your manuscripts at

https://www.hindawi.com
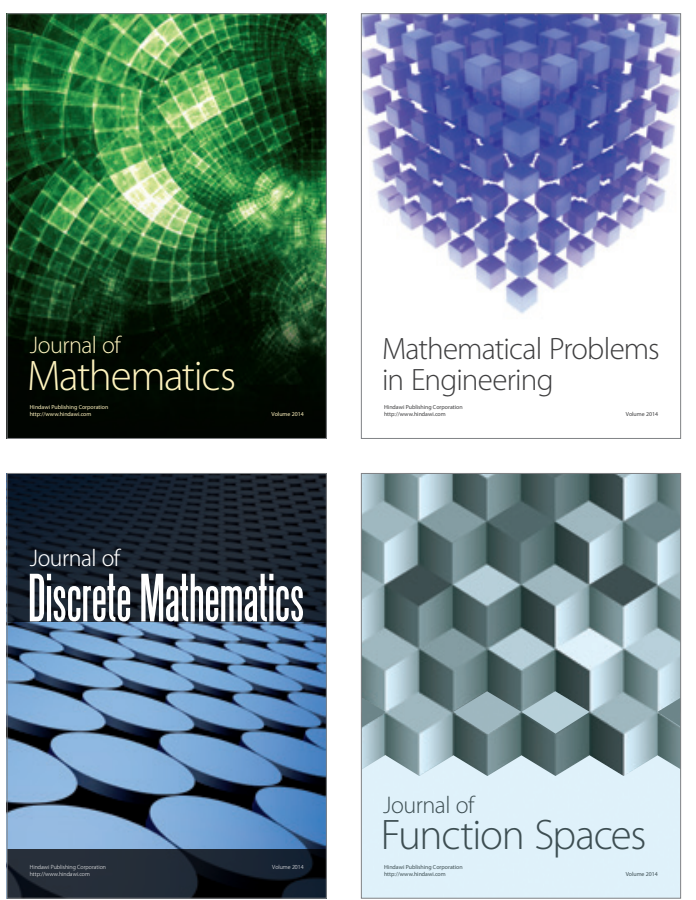

Mathematical Problems in Engineering
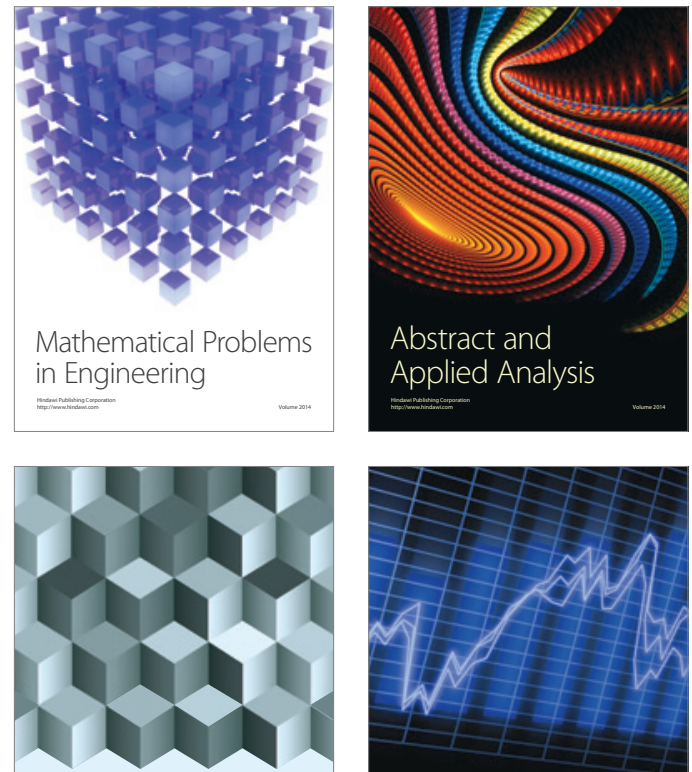

Journal of

Function Spaces

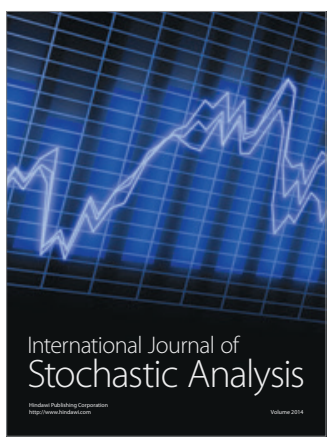

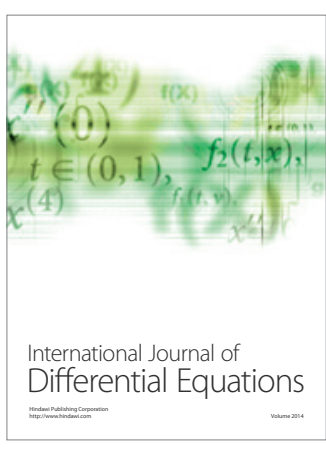
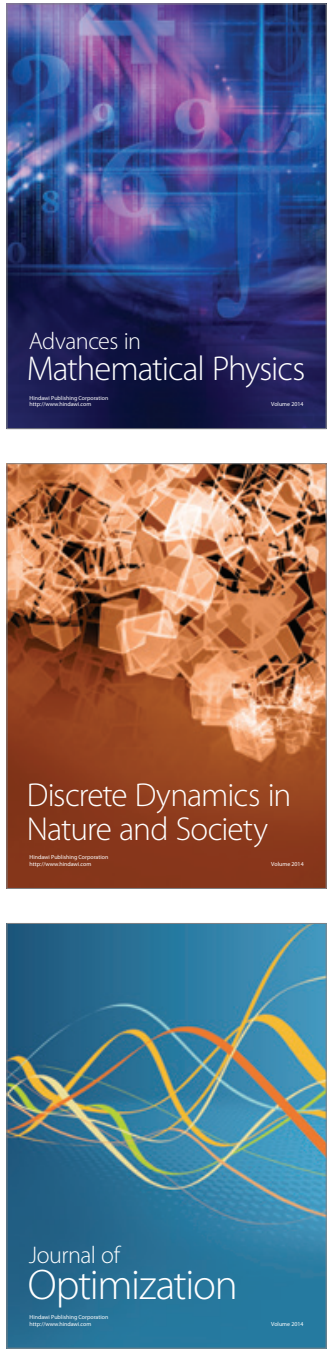